\title{
TÁC ĐỘNG CỦA CÁC YẾU TỐ HÀNH VI ĐẾN QUYẾT ĐỊNH VÀ HIÊU QUẢ ĐẦU TƯ CỦA NHÀ ĐẦU TƯ CÁ NHÂN TRÊN THỊ TRƯỜNG CHÚNG KHOÁN THÀNH PHỐ HỒ CHÍ MINH
}

\author{
Võ Thị Hiếu ${ }^{1}$, Bùi Hũu Phước², Bùi Nhất Vương ${ }^{3}$
}

\section{TÓM TĂT}

Mục tiêu của nghiên cứu này là để khám phá các nhân tố hành vi ảnh hưởng đến quyết định đầu tu và hiệu quả đầu tur của nhà đầu tu cá nhân. Dũ liệu khảo soát đã thu thập tù̀ 411 nhà đầu tư cá nhân tại thị trương chưng khoán Tp. HCM để cung cấp bằng chứng. Kết quả tùu phân tích hồi quy bội bởi sư dựng phần mềm SPSS 20.0 đã cho thấy rằng Neo quyết định, Sự quá tư tin, Khuynh hướng sẵn có, Hiệu ứng đám đông, Tình huống điển hình đã liên kết tích cưc với quyết định đầu tur. Tuy nhiên, quyết định đầu tu được thúc đẩy bởi các nhân tố này ảnh hưởng tiêu cực đến hiệu quả đà̀u tu. Bên cạnh đó, nghiên cúu cũng đã chỉ ra rằng nhũng nhà đầu tu nam có kinh nghiệm đầu tu có khuynh huớng thu được hiệu quả đầu tu cao hơn nhũng nhà đầu tu nũ ít kinh nghiệm và nhũng nhà đầu tư càng lớn tuổi có khuynh huớng đầu tu ít hiệu quả hơn các nhà đầu tu trẻ tuổi. Những phát hiện chính của nghiên cứu này cung cấp các hàm ý thực tiễn cho đầu tu cá nhân. Nó ngứy rằng các nhà đầu tư cá nhân nên nên cải thiện 05 yếu tố hành vi này để gia tăng hiệu quả đầu tu của ho trên thị truờng chúng khoán TP. HCM.

Tù khóa: Neo quyết định, Sự quá tụ tin, Khuynh hương sã̃n có, Hiệu ứng đám đông, Tình huống điển hình, hiệu quả đầu tu

\section{THE EFFECT OF BEHAVIORAL FACTORS ON INDIVIDUAL INVESTORS' DECISION MAKING AND INVESTMENT PERFORMANCE AT THE HO CHI MINH STOCK EXCHANGE}

\begin{abstract}
The objective of this study is to explore the behavioral factors that influence individual investors' investment decisions and investment performance. Survey data was collected from 411 individual investors in the Ho Chi Minh City Stock Exchange to provide evidence. Results from multiple regression analyses using SPSS 20.0 software revealed that Anchoring, Overconfidence, Availability, Herding effect, and Representativeness positively associated with investment decisions. However, investment decisions were driven by these factors negatively affected investment performance. Besides, the study also showed that male investors with trading experience tended to obtain a higher return on investment than less experienced female investors and older investors were less effective than young investors. The main findings of this study provided practical implications for individual investors. It implied that individual investors should improve these five behavioral factors to increase their investment performance in the HCMC stock market.
\end{abstract}

Keywords: anchoring, overconfidence, availability, herding effect, representativeness, investment performance

\footnotetext{
${ }^{1}$ Học viên Cao học, Trường Đại học Tài chinh - Marketing. E-mail: hieuquangngai1 @ gmail.com, Điện thoại di động: 0983956178

2 TS. Bùi Hữu Phước, Trường Đại học Tài chính - Marketing. E-mail: phuoctcnh@ufm.edu.vn, ĐT: 0913100394

${ }^{3}$ TS. Bùi Nhất Vương, Khoa Vận tải Hàng không, Học Viện Hàng Không Việt Nam. E-mail: nhatvuonga1 @ gmail.com, ĐT: 0908580139
} 


\section{GIỚI THIÊU}

Thị trường chứng khoán là một thị trường tại đó cổ phiếu được mua và bán (Zuravicky, 2005). Trong một nền kinh tế, bên cạnh vai trò là nguồn đầu tư tài chính, thị trường chứng khoán còn thực hiện chức năng như một cơ chế báo hiệu cho các nhà quản lý về các quyết định đầu tư và là chất xúc tác cho quản trị doanh nghiệp. Bên cạnh đó, thị trường chứng khoán được biết đến là kênh hiệu quả nhất để tăng vốn của công ty (Zuravicky, 2005). Mọi người quan tâm đến chứng khoán vì sự tăng trưởng dài hạn của vốn, cổ tức và hàng rào chống lại sự xói mòn lạm phát của sức mua. Tính năng khác làm cho thị trường chứng khoán hấp dẫn hơn các loại hình đầu tư khác là tính thanh khoản của nó.

Có thể coi thị trường chứng khoán là thước đo cho sức mạnh và sự phát triển kinh tế. Do đó, sự chuyển động của xu hướng thị trường chứng khoán đại diện cho sức khỏe kinh tế của một nền kinh tế. Việc tăng giá cổ phiếu có xu hướng liên quan đến sự gia tăng đầu tư, dẫn đến tốc độ tăng trưởng cao hơn của một công ty nói riêng và một nền kinh tế nói chung. Ngoài ra, thị trường chứng khoán có thể thúc đẩy sự phát triển của một nền kinh tế thông qua việc phân bổ nguồn lực hiệu quả và sử dụng tốt hơn các nguồn lực (Qamruzzaman \& Wei, 2018). Do đó, tầm quan trọng và ảnh hưởng của thị trường chứng khoán đối với sự phát triển của một nền kinh tế không thể phủ nhận. Mục đích xây dựng thị trường chứng khoán Việt Nam không nằm ngoài những mục đích nêu trên. Chính phủ Việt Nam chủ trương thành lập thị trường chứng khoán để hỗ trợ doanh nghiệp huy động vốn dài hạn cho sản xuất và kinh doanh (HOSE, 2010). Tuy nhiên, tại thời điểm thành lập, thị trường chứng khoán vẫn còn là một điều gì đó quá xa lạ và mơ hồ đối với hầu hết người dân Việt Nam (HOSE, 2010). Do đó, việc xây dựng thị trường chứng khoán dường như bắt đầu từ không đủ nền tảng pháp lý, hệ thống giao dịch đơn giản và rất ít công ty chứng khoán và các loại chứng khoán hạn chế (HOSE, 2010). Ở giai đoạn thành lập, năm 2000, thị trường chứng khoán Việt Nam chỉ có 2 công ty niêm yết và 4 công ty chứng khoán. Đã trải qua rất nhiều thăng trầm, hiện tại, nó có hai trung tâm giao dịch; một trong số đó là Sở giao dịch chứng khoán Hồ Chí Minh cho các công ty có vốn từ 80 tỷ đồng. Đến tháng 10 năm 2019, Sàn giao dịch chứng khoán Hồ Chí Minh có khoảng 404 cổ phiếu được niêm yết (Stockbiz, 2019). Tuy nhiên, so với thị trường chứng khoán nước ngoài, thị trường chứng khoán Việt Nam dường như nhỏ hơn nhiều về quy mô và sự trưởng thành. Nghiên cứu này tập trung vào sự phát triển của sàn giao dịch, được đánh giá thông qua VN-Index.

Mặc dù thị trường chứng khoán Hồ Chí Minh đã được phát triển đáng kể cả về số lượng cổ phiếu niêm yết và giá trị giao dịch trong 19 năm qua, sự biến động giá dường như biến động khó lường qua các giai đoạn khác nhau (Hình 1) và sự hiểu biết về hành vi của các nhà đầu tư cá nhân và các yếu tố hành vi ảnh hưởng đến quyết định đầu tư của họ là rất hạn chế. Các yếu tố hành vi là các yếu tố tâm lý bao gồm cảm xúc và nhận thức, đóng vai trò quan trọng trong quá trình ra quyết định của các nhà đầu tư (Waweru và cộng sự., 2008). Những lý thuyết này cho rằng các nhà đầu tư lý trí tối đa hóa lợi nhuận của họ bằng cách tuân theo các quy tắc tài chính cơ bản và đưa ra quyết định đầu tư về việc cân nhắc lợi nhuận. Tuy nhiên, mức độ chấp nhận rủi ro của các nhà đầu tư phụ thuộc vào đặc điểm cá nhân và thái độ của họ đối với rủi ro (Maditinos và cộng sự., 2017). Do đó, cần phải tìm hiểu các yếu tố hành vi tác động đến quá trình ra quyết định của các nhà đầu tư cá nhân trên thị trường chứng khoán Hồ Chí Minh hiện tại để giúp các nhà đầu tư cũng như các công ty chứng khoán đưa ra dự đoán và quyết định tốt hơn cho kinh doanh của họ. 


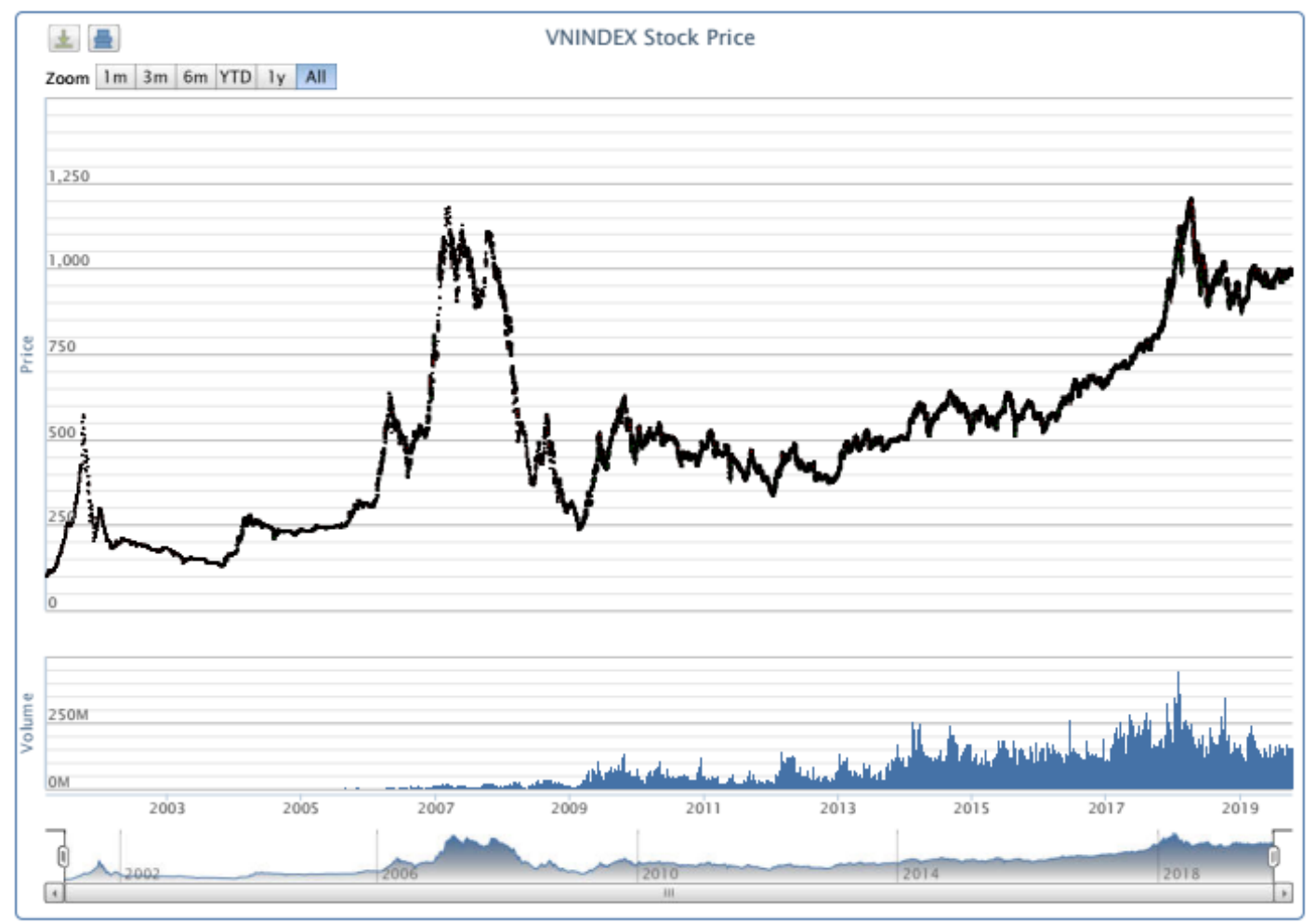

Hình 1. VN-Index từ năm 2000 to 2019

(Nguồn: Vndirect, 2019)

Hơn nữa, các nghiên cứu trước đây về việc ra quyết định đầu tư đã cung cấp các hướng dẫn phù hợp cho các nhà đầu tư để đưa ra quyết định sáng suốt tại các nước phát triển (Dima và cộng sự., 2018). Do đó, cần kiểm tra các yếu tố ảnh hưởng đến việc ra quyết định của nhà đầu tư trong một môi trường đang phát triển như Việt Nam bởi vì kiến thức về tác động tiềm tàng của các yếu tố đó giúp các nhà quản lý đưa ra quyết định sáng suốt trong tình hình biến động cao của giá cổ phiếu (Greenberg \& Hershfield, 2019). Bên cạnh đó, Dima và cộng sự. (2018) cũng tranh luận rằng các quyết định đầu tư phù hợp có giúp các nhà đầu tư cá nhân gia tăng tích cực đến hiệu quả đầu tư của họ. Do đó, nghiên cứu này nhằm cải thiện việc ra quyết định và hiệu quả đầu tư của các nhà đầu tư cá nhân bằng cách điều tra "tác động của các yếu tố hành vi đến quyết định và hiệu quả đầu tu của nhà đầu tu cá nhân trên thị truờng chứng khoán
Thành phố Hồ Chí Minh" là thực sự cần thiết.

\section{CƠ SỞ LÝ THUYẾT}

\section{1. Định nghĩa về tài chính hành vi}

Trong những nghiên cứu hiện nay thì tài chính hành vi đã trở thành một phần không thể thiếu đến các quyết định đầu tư của nhà đầu tư cá nhân, ảnh hưởng không nhỏ đến hiệu quả của hoạt động đầu tư (Kengatharan \& Navaneethakrishnan, 2014). Tài chính hành vi là một lĩnh vực tài chính đưa ra các lý thuyết đựa trên các phân tích tâm lý để giải thích những diễn biến bất thường trên thị trường chứng khoán. Tài chính hành vi thường dựa trên giả định rằng cấu trúc thông tin và đặc tính của những người tham gia vào thị trường chứng khoán đều bị ảnh hưởng một cách hệ thống bởi các quyết định đầu tư cá nhân cũng như các tác động của thị trường. Lý thuyết tài chính hành vi chỉ ra rằng cơ chế điều chỉnh thị trường về trạng thái cân bằng không phải lúc nào cũng có thể xảy ra. Có nghĩa là sẽ có 
trường hợp những nhà đầu tư "hợp lý" sẽ không thể chiến thắng những nhà đầu tư "bất hợp lý". Lúc này thị trường sẽ không hiệu quả, hay tài sản tài chính được định giá quá cao hoặc quá thấp. Đã có rất nhiều nghiên cứu chỉ ra các hiện tượng đã xảy ra trong thị trường chứng khoán đối lập với thuyết thị trường hiệu quả, không thể giải thích được một cách thoả đáng theo mô hình này nhưng lại có thể lý giải dựa trên các lý thuyết tài chính hành vi.

Theo Bikas và cộng sự. (2013), hiện nay có nhiều quan niệm khác nhau về tài chính hành vi nhưng chủ yếu chỉ ra tài chính hành vi nghiên cứu ảnh hưởng của tâm lý lên hành vi của những người tham gia trên thị trường tài chính và kết quả là những ảnh hưởng đó lên thị trường. Như vậy hành vi tài chính là một cách hiểu mới về tài chính giúp bổ sung vào các lý thuyết tài chính cơ bản bằng cách giải thích hành vi của quá trình ra quyết định. Ngược lại với lý thuyết của Markowitz (1952) và Sharpe (1964), hành vi tài chính giải quyết vấn đề của các cá nhân và cách mà họ thu thập và sử dụng thông tin. Hành vi tài chính tìm cách để hiểu và dự đoán được quá trình ra quyết định có quan hệ đến hệ thống thị trường tài chính. Ngoài ra, nó còn tập trung vào việc áp dụng các nguyên lý tâm lý và kinh tế để cải thiện việc ra quyết định tài chính (Olsen, 1998). Tài chính hành vi được định nghĩa như là một lĩnh vực tài chính để giải thích những khác thường trên thị trường chứng khoán bằng việc sử dụng những thành kiến tâm lý hơn là bỏ qua những kết quả ngẫu nhiên phù hợp với giả thuyết thị trường hiệu quả (Fama, 1998). Nó được giả định rằng các nhà đầu tư cá nhân và các kết quả của thị trường thì bị ảnh hưởng bởi các cấu trúc thông tin, các đặc điểm tính cách khác nhau của những người tham gia thị trường (Bakar \& Yi, 2016).

\subsection{Tài chính hành vi ở châu á}

Việt Nam là một nền kinh tế mới nổi ở châu Á với nhiều đặc điểm văn hóa tương tự như các nước châu Á khác. Phần này sẽ cung cấp một cái nhìn tổng quan về tài chính hành vi ở châu Á và tầm quan trọng của tài chính hành vi ở châu Á nói chung và ở Việt Nam nói riêng.

Châu Á được biết đến với mức độ đa dạng về thị trường vốn và kinh nghiệm tài chính người tham gia, vì vậy đây là một nơi thú vị để nghiên cứu tài chính hành vi. Mặc dù một số nền kinh tế vẫn đang ở giai đoạn phát triển, một số nền kinh tế khác đã được phát triển trong một thời gian dài. Vì trình độ kiến thức và kinh nghiệm khác nhau dẫn đến sự khác biệt trong việc ra quyết định, Châu Á là một nền tảng hoàn hảo để nghiên cứu tài chính hành vi. Hơn nữa, người châu Á dường như bị thiên kiến nhận thức nhiều hơn người phương Tây và các nhà đầu tư cá nhân châu Á được coi là những nhà đầu tư đơn thuần (Kim \& Nofsinger, 2008). Về mặt lý thuyết, các nhà khoa học xã hội và nhà tâm lý học tin rằng xu hướng thiên về hành vi có thể được nuôi dưỡng bởi văn hóa mặc dù mức độ có thể khác nhau (Yates và cộng sự., 1997). Kim và Nofsinger (2008) giải thích sự khác biệt giữa các nền văn hóa thông qua chủ nghĩa tập thể và chủ nghĩa cá nhân. Các nền văn hóa châu Á được cho là thuộc về mô hình tập thể xã hội, điều này đã gây ra các nhà đầu tư trực tiếp quá tự tin dẫn đến sự thiên lệch về hành vi. Sự khác biệt về văn hóa, cụ thể hơn, kinh nghiệm sống và giáo dục có thể ảnh hưởng đến hành vi, do đó, các nhà nghiên cứu cho rằng khuynh hướng hành vi có thể khác nhau giữa các nền văn hóa khác nhau. Một số bằng chứng đã được tìm thấy để chứng minh rằng người châu Á thể hiện nhiều thành kiến hành vi hơn những người được nuôi dưỡng ở các nước phương Tây (Yates và cộng sự., 1997). Mặc dù có một số tài liệu về sự khác biệt về hành vi giữa người châu Á và người phương Tây, nhưng những tài liệu này vẫn còn khan hiếm (Kim \& Nofsinger, 2008). Theo Weber và Hsee (2010), điểm mấu chốt là chủ đề văn hóa và ra quyết định không nhận được nhiều sự quan tâm của các nhà nghiên cứu hoặc nhà tâm lý học đa văn hóa. Ngoài ra, một tài liệu có hệ thống về hành vi của 
người châu Á và ảnh hưởng của chúng đối với việc ra quyết định đầu tư được cung cấp bởi Chen và cộng sự. (2017). Để hỗ trợ cho lý thuyết này, họ thấy rằng các nhà đầu tư Trung Quốc phải chịu sự thiên vị quá mức và ảnh hưởng đến quyết định nhiều hơn so với các nhà đầu tư Hoa Kỳ (Kim \& Nofsinger, 2008). Mặc dù tài chính hành vi vẫn là một chủ đề gây tranh cãi, các nhà phân tích tài chính hiện đã hiểu rõ hơn về hành vi của con người và chấp nhận rằng những hành vi này có thể ảnh hưởng đến quyết định tài chính. Nhiều nhà nghiên cứu cũng đồng ý rằng chênh lệch giá bị hạn chế (Shleifer \& Vishny, 2017), do đó, những hành vi này có thể ảnh hưởng đến giá cả. Trong khi đó, các nghiên cứu về tài chính hành vi đã nâng cao kiến thức về thị trường tài chính. Gần đây, các phiên thảo luận về tài chính hành vi trong các hội nghị tài chính dường như có nhiều người tham dự là những học giả trẻ được đào tạo từ chuyên ngành tài chính (Kim \& Nofsinger, 2008). Điều này tạo ra triển vọng hơn trong tương lai về nghiên cứu tài chính hành vi.

\subsection{Lý thuyết về tài chính hành vi}

Fernandes và cộng sự. (2007) đã phân chia các khuynh hướng hành vi ra làm hai nhóm: khuynh hướng nhận thức và khuynh hướng cảm xúc, mặc dù cả hai loại đều mang lại những quyết định bất hợp lý. Bởi vì khuynh hướng nhận thức (sự tự nghiệm) như việc neo quyết định, sự sẵn có, và tình huống điển hình bắt nguồn từ sự suy luận không chính xác, những thông tin và lời khuyên tốt hơn thường có thể hiệu chỉnh chúng. Trái lại, những khuynh hướng cảm xúc, chẳng hạn như sự tiếc nuối và sợ rủi ro, bắt nguồn từ những cảm giác bốc đồng hoặc trực giác, hơn là từ suy luận có ý thức, và khó có thể hiệu chỉnh.

Khuynh hướng nhận thức (sự tự nghiệm) nói đến quy luật ngón tay cái mà con người sử dụng để ra quyết định trong những môi trường phức tạp, không chắc chắn. Khuynh hướng cảm xúc có thể tác động đến quá trình ra quyết định, được tập hợp một cách thuận lợi theo Lý Thuyết Triển Vọng (Kahneman \& Tversky, 1979). Lý thuyết này đề ra một khuôn khổ mô tả về cách người ta ra quyết định dưới điều kiện rủi ro và không chắc chắn và bao gồm một khuôn khổ về hành vi phong phú hơn lý thuyết hữu dụng kỳ vọng chủ quan dưới nhiều mô hình kinh tế (Masomi \& Ghayekhloo, 2011).

\section{GIẢ THUYÊT NGHIÊN CỨU}

Nghiên cứu này lựa chọn mô hình dựa vào các lý thuyết tài chính hành vi như: Lý thuyết sự tự nghiệm, Lý thuyết triển vọng. Ngoài ra, nghiên cứu dựa vào các nghiên cứu thực nghiệm về ảnh hưởng của tài chính hành vi quyết định đầu tư cá nhân đã được một số tác giả nghiên cứu như: Bakar và Yi (2012), Abul (2019). Trên nền tảng cùng là nghiên cứu quyết định đầu tư của nhà đầu tư cá nhân, đây sẽ là cơ sở lý thuyết vững chắc, giúp nghiên cứu vận dụng mô hình phù hợp với điều kiện nghiên cứu tại sở giao dịch chứng khoán thành phố Hồ Chí Minh. Như vậy, từ cơ sở lý thuyết nền, các mô hình nghiên cứu trong và ngoài nước trước đây, và từ kết quả nghiên cứu định tính, tác giả lựa chọn mô hình nghiên cứu các yếu tố tài chính hành vi ảnh hưởng đến quyết định đầu tư của nhà đầu tư cá nhân, gồm 5 yếu tố: (1) Sự quá tự tin, (2) Khuynh hướng sẵn có, (3) Tình huống điển hình, (4) Hiệu ứng đám đông, (5) Neo quyết định (Hình 2).

\subsection{Sự quá tự tin}

Sự quá tự tin mô tả trạng thái tâm lý mà các nhà đầu tư tin rằng họ sở hữu nhiều kiến thức hơn các nhà đầu tư khác (Shiller, 2005). Hiện tượng quá tự tin đã được nghiên cứu bởi nhiều nhà nghiên cứu, như là Bakar và Yi (2016), và Longjie và Anfeng (2017). Các nhà đầu tư quá tự tin tin rằng họ có thể kiếm được nhiều hơn từ thị trường bằng cách sử dụng cảm xúc của mình, mặc dù điều này là không thể. Tuy nhiên, các nhà đầu tư ích kỷ, những người coi mình là thiên tài do biết khi nào là thời điểm tốt nhất để giao dịch và để thu được lợi nhận nhanh 
chóng từ thị trường chứng khoán, nhưng mà họ không thể đánh bại thị trường như họ giả định. Odean (1998) lập luận rằng các nhà đầu tư quá tự tin tin rằng họ thông minh hơn các nhà đầu tư khác liên quan đến việc chọn thời điểm tốt nhất để mua cổ phiếu với giá tốt nhất. Bakar và Yi (2016) thấy rằng các quyết định của các nhà đầu tư bị ảnh hưởng đáng kể bởi sự tự tin thái quá. Hơn nữa,
Longjie và Anfeng (2017) tìm thấy mối tương quan tích cực giữa sự tự tin thái quá và mức đầu tư vào thị trường chứng khoán. Dựa vào những thảo luận trên, tác giả đề xuất giả thuyết như sau:

$H_{1}$ : Sự quá tụ tin ảnh hương thuận chiều đến quyết định đầu tu của nhà đầu tu cá nhân.

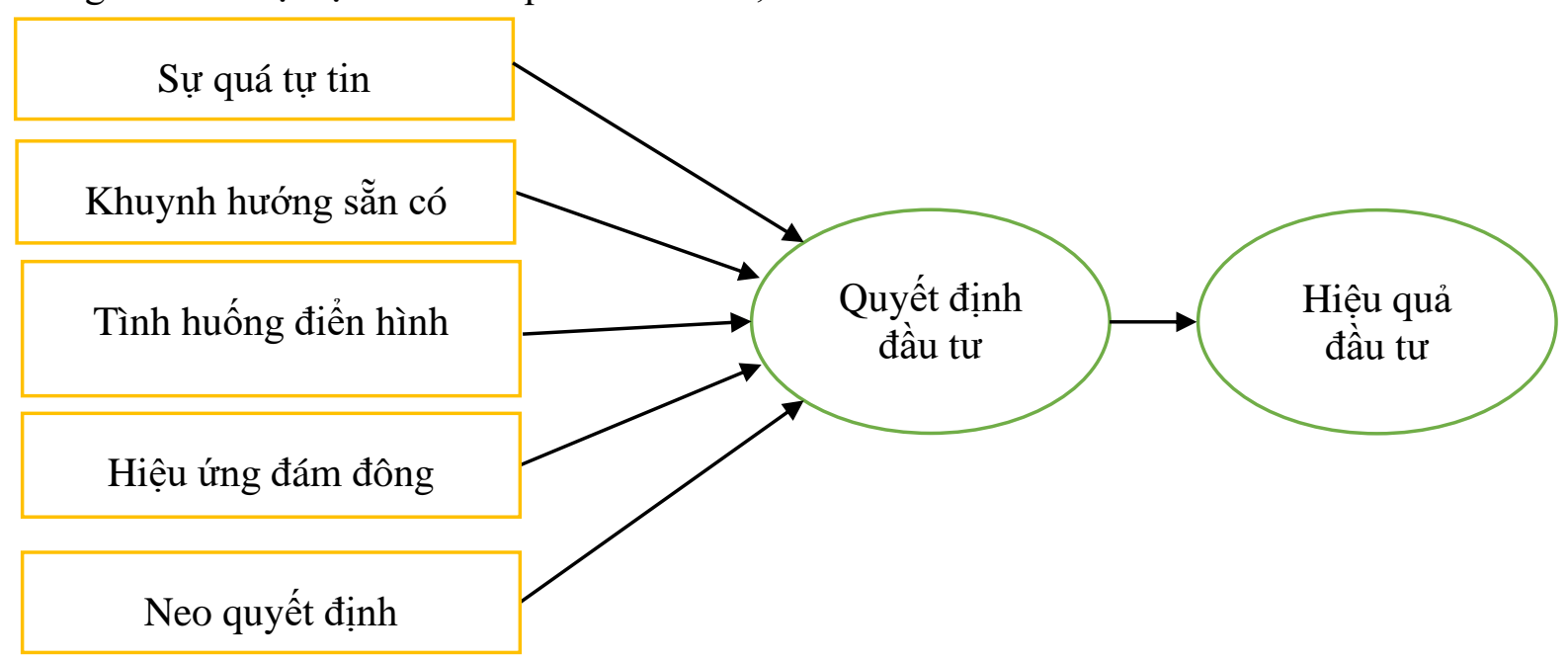

Hình 2. Mô hình nghiên cứu các yếu tố tài chính hành vi ảnh hưởng đến quyết định và hiệu quả đầu tư của nhà đầu tu cá nhân tại sở chứng khoán TP. HCM

(Nguồn: tác giả nghiên cưu đề xuất)

\subsection{Khuynh hướng sẵn có}

Theo Shefrin và Statman (2000), khuynh hướng sẵn có xảy ra do các nhà đầu tư dự vào thông tin để đưa ra quyết định sáng suốt, nhưng không phải tất cả thông tin đều có sẵn. Các nhà đầu tư có xu hướng tăng thêm tầm quan trọng của nhiều thông tin có sẵn hơn và giảm sự chú ý đối với thông tin được chú ý ít thường xuyên hơn. Nói chung, mọi người dễ dàng nhớ lại các thông tin đã xuất hiện gần đây, đặc biệt là trên các phương tiện truyền thông và các bản phát hành của công ty, bởi vì các khuyến nghị của nhà môi giới hoặc cố vấn của họ rất mới mẻ trong bộ nhớ của họ. Một số nhà nghiên cứu đã cho rằng khuynh hướng sẵn có tác động tích cực đến quyết định tư của các nhà đầu tư các nhân (Bakar \& Yi, 2016). Do đó, tác giả đề xuất giả thuyết như sau:
$\mathrm{H}_{2}$ : Khuynh hwớng sã̃n có ảnh huởng thuận chiều đến quyết định đầu tu của nhà đầu tu cá nhân.

\subsection{Tình huống điển hình}

Một ví dụ điển hình cho "Tình huống điển hình" là các nhà đầu tư thường suy ra một công ty có tốc độ tăng trưởng dài hạn cao sau một số quý tăng (Waweru và cộng sự., 2008). Tình huống điển hình (khuynh hướng đại diện) cũng dẫn đến cái gọi là bỏ qua kích thước mẫu, nó xảy ra khi mọi người cố gắng suy luận từ cỡ mẫu quá nhỏ (Barberis \& Thaler, 2003). Trong thị trường chứng khoán, khi các nhà đầu tư tìm cách mua cổ phiếu của nhóm "hot", điều này có nghĩa là khuynh hướng đại diện được áp dụng. Hành vi này là một lời giải thích cho phản ứng thái quá của nhà đầu tư (De Bondt \& Thaler, 1985). Các cổ phiếu tốt được xem là cổ phiếu của các công ty tốt và các công ty tốt 
lại được xem là công ty an toàn, điều này một lần nữa phù hợp với hoạt động của khuynh hướng đại diện. Nhiều nhà đầu tư dường như tin rằng nếu giá đã tăng trong quá khứ thì chúng sẽ tiếp tục tăng, và ngược lại với giá giảm. Khái niệm về Khuynh hướng đại diện cho thấy rằng điều này là do những nhà đầu tư đó nhìn thấy một khoản đầu tư với mức tăng giá gần đây là đại diện cho các khoản đầu tư thành công dài hạn, ngược lại với việc giảm giá. De Bondt và Thaler (1985) lập luận rằng vì các nhà đầu tư chịu sự thiên vị về tính đại diện, họ có thể trở nên quá lạc quan về những người chiến thắng trong quá khứ và quá bi quan về những người thua cuộc trong quá khứ. Do đó, tác giả đề xuất giả thuyết như sau:

$H_{3}$ : Tình huống điển hình ảnh huởng thuận chiều đến quyết định đầu tu của nhà đầu tu cá nhân.

\subsection{Hiệu ứng đám đông}

Hiệu ứng đám đông là tại đó các nhà đầu tư cá nhân theo xu hướng của thị trường. Do đó, thay vì sử dụng thông tin của chính họ khi đưa ra quyết định, họ chỉ cần làm theo những gì các nhà đầu tư khác làm. Trên thực tế, nhiều nhà đầu tư có thể không phản hồi tức thì với thông tin mới mà có thể dựa trên hành động của họ đối với hoạt động giao dịch của những nhà đầu tư được cho là có nhiều thông tin. Bakar và Yi (2016) lập luận rằng hiệu ứng đám đông xảy ra khi các nhà đầu tư tìm đến hành vi của các nhà đầu tư khác vì họ tin rằng những người khác sở hữu nhiều thông tin hơn. Ông lập luận rằng, trong một môi trường ảnh hưởng bởi tâm lý đám đông, thì một đoạn tin tức có thể dẫn đến một sự thay đổi lớn về giá. Điều này có thể khiến tất cả những người tham gia vào thị trường chứng khoán đưa ra quyết định sai lầm. Chiang và Zheng (2010) thấy rằng hiệu ứng đám đông có tác động tiêu cực đến thị trường chứng khoán, vì nó có thể gây ra biến động, bong bóng và chứng khoán bị đánh giá quá thấp. Tác động của hiệu ứng đám đông cũng có thể ảnh hưởng tiêu cực đến cơ chế cung và cầu của thị trường. Abul
(2019) phát hiện ra rằng các nhà đầu tư nhỏ tham gia vào thị trường chứng khoán có $x u$ hướng tuân theo hiệu ứng đám đông. Do đó, tác giả đề xuất giả thuyết như sau:

H4: Hiệu ưng đám đông ảnh huởng thuận chiều đến quyết định đầu tu của nhà đầu tu cá nhân.

\subsection{Neo quyết định}

Trong quá trình ra quyết định, con người có xu hướng dựa vào thông tin trong tay, được gửi từ gia đình hoặc bất kỳ nguồn khuyến nghị nào khác (Singha \& Sunita, 2015) thay vì thông tin mới. Trong trường hợp đầu tư chứng khoán, đó là hành vi của cá nhân, người nhớ lại giá của cổ phiếu khi họ tham gia đầu tư lần đầu tiên thay vì giá trị của cổ phiếu sau một thời gian. Các nhà đầu tư bị ảnh hưởng bởi neo quyết định trong khi đáng lẽ phải ước tính lợi nhuận của công ty (Cen và cộng sự., 2013). Nói cách khác, neo quyết định xảy ra khi một quy mô giá trị được cố định hoặc neo chặt bởi những quan sát gần đây. Điều này dẫn các nhà đầu tư tới việc kỳ vọng một cổ phần sẽ tiếp tục giao dịch trong một biên độ được xác phù hợp với xu hướng lịch sử. Do đó, tác giả đề xuất giả thuyết như sau:

H5: Neo quyết định ảnh huởng thuận chiều đến quyêt định đầu tu của nhà đầu tu cá nhân.

\subsection{Quyết định đầu tư và hiệu quả đầu tư}

Anderson và cộng sự. (2005) đã kết luận rằng các nhà đầu tư cá nhân thực hiện số lượng giao dịch cao hơn có thể mang lại lợi nhuận cao hơn so với các cá nhân có ít giao dịch hơn. Kourtidis (2017) tuyên bố rằng các cổ phiếu có mức tăng sở hữu cá nhân lớn nhất có thể kiếm được lợi nhuận bất thường tiêu cực trong năm; trong khi đó, các cổ phiếu giảm nhiều nhất sở hữu cá nhân có thể kiếm được lợi nhuận bất thường tích cực. Họ cũng đi sâu hơn về hành vi mua và bán và nghiên cứu hiệu quả trong quá khứ của các cổ phiếu đã mua và bán này. Các tác giả nhận thấy rằng các cổ phiếu có sự gia tăng đáng kể về sở hữu cá nhân (cổ phiếu đã 
mua) là các cổ phiếu chiến thắng trong quá khứ. Những kết quả như vậy đã dẫn đển giả thuyết rằng hiệu quả có liên quan tích cực đến tính toàn diện / hợp lý và chính thức hóa trong quá trình ra quyết định đầu tư. Có thể thấy rằng mối quan hệ giữa việc ra quyết định và hiệu quả của các nhà đầu tư cá nhân được đề xuất trong một khoảng thời gian tại Việt Nam, tuy nhiên, không có nhiều nghiên cứu thực nghiệm để chứng minh liệu rằng các nhà đầu tư cá nhân ra quyết định có tác động đến hiệu quả của nhà đầu tư hay không. Do đó, một giả thuyết được đề xuất như sau:

H6: Quyết định đầu tu do ảnh huởng bởi hành vi có thể tác động ngược chiều đến hiệu quả đầu tư của nhà đầu tư cá nhân.

\section{KẾT QUẢ NGHIÊN CÚU}

Nghiên cứu được thực hiện với đối tượng khảo sát là nhà đầu tư cá nhân giao dịch tại sở chứng khoán TP.HCM. Tổng cộng có 424 bảng câu hỏi được phát ra, thu về 424 bảng, loại ra 13 bảng không đạt yêu cầu, còn lại 411, đạt tỷ lệ 96,93\%. Bảng câu hỏi được mã hoá và đưa vào xử lý số liệu bằng phần mềm SPSS 20.0 để phân tích. Phân loại 411 người tham gia trả lời theo thành phần giới tính, độ tuổi, kinh nghiệm, và trình độ học vấn khi được đưa vào xử lý (Bảng 1).

Bảng 1. Đặc điểm nhà đầu tư

\begin{tabular}{|c|c|c|c|}
\hline & & Tần số & Phần trăm (\%) \\
\hline \multirow{2}{*}{ Giới tính } & Nũ & 254 & 61.8 \\
\hline & Nam & 157 & 38.2 \\
\hline \multirow{4}{*}{ Độ tuổi } & $<25$ & 74 & 18.0 \\
\hline & Từ 26-35 & 181 & 44.0 \\
\hline & Từ 36-45 & 97 & 23.6 \\
\hline & Trên 45 & 59 & 14.4 \\
\hline \multirow{4}{*}{ Kinh nghiệm đầu tư } & < 1 năm & 33 & 8.0 \\
\hline & 1-3 năm & 190 & 46.2 \\
\hline & 3-5 năm & 152 & 37.0 \\
\hline & $>5$ năm & 36 & 8.8 \\
\hline \multirow{4}{*}{ Trình độ học vấn } & Dưới đại học & 107 & 26.0 \\
\hline & Đại học & 209 & 50.9 \\
\hline & Thạc sĩ & 87 & 21.2 \\
\hline & Tiến sĩ & 8 & 1.9 \\
\hline \multicolumn{2}{|c|}{ Tổng } & 411 & 100.0 \\
\hline
\end{tabular}

Kết quả nghiên cứu cho thấy tỷ lệ trả lời đã cho thấy có sự chênh lệch lớn về giới tính. Nam giới có xu hướng tham gia thị trường chứng khoán nhiều hơn nữ giới và các nhà đầu tư cá nhân có độ tuổi khá trẻ tập trung vào khoảng 26-35 tuổi.

\section{1. Độ tin cậy và tính giá trị của thang đo}

Thang đo trước hết sẽ được phân tích hệ số tin cậy Cronbach's Alpha, các biến có hệ số tương quan tổng nhỏ hơn 0,3 sẽ bị loại và thang đo được chấp nhận để phân tích trong các bước tiếp theo khi có độ tin cậy Cronbach Alpha từ 0,7 trở lên (Giao \& Vương, 2019). Kết quả phân tích từ Bảng 2 cho thấy hệ số Cronbach's Alpha của thang đo giao động trong phạm vi từ 0,794 đến 0,909 và hệ số tương quan biến tổng nhỏ nhất của các biến quan sát trong thang đo là 0,614 . Nhìn chung, các thang đo trên đều có hệ số Cronbach's Alpha khá cao $(>0,8)$ và tất cả các biến quan sát của thang đo này đều có hệ số tương quan biến tổng lớn hơn 
0,3 do đó chúng đều đạt được độ tin cậy rất tốt.

Bảng 2. Kết quả phân tích độ tin cậy và tính giá trị của thang đo

\begin{tabular}{|c|c|c|c|c|}
\hline Thang đo & $\begin{array}{c}\text { Biến } \\
\text { quan sát }\end{array}$ & $\begin{array}{c}\text { Hệ số } \\
\text { tải nhân } \\
\text { tố }\end{array}$ & $\begin{array}{l}\text { Cronbach's } \\
\text { alpha }\end{array}$ & $\begin{array}{c}\text { Hệ số tương } \\
\text { quan biến- tống } \\
\text { nhỏ nhất }\end{array}$ \\
\hline \multirow{5}{*}{ Sự quá tự tin } & QTT2 & 0,798 & \multirow{5}{*}{0,843} & \multirow{5}{*}{0,614} \\
\hline & QTT3 & 0,788 & & \\
\hline & QTT1 & 0,767 & & \\
\hline & QTT5 & 0,753 & & \\
\hline & QTT4 & 0,723 & & \\
\hline \multirow{3}{*}{ Khuynh hướng sẵn có } & KHSC3 & 0,856 & \multirow{3}{*}{0,829} & \multirow{3}{*}{0,684} \\
\hline & KHSC2 & 0,843 & & \\
\hline & KHSC1 & 0,749 & & \\
\hline \multirow{4}{*}{ Tình huống điển hình } & THDH2 & 0,856 & \multirow{4}{*}{0,865} & \multirow{4}{*}{0,682} \\
\hline & THDH4 & 0,847 & & \\
\hline & THDH3 & 0,842 & & \\
\hline & THDH1 & 0,823 & & \\
\hline \multirow{4}{*}{ Hiệu ứng đám đông } & HUDD2 & 0,852 & \multirow{4}{*}{0,869} & \multirow{4}{*}{0,702} \\
\hline & HUDD4 & 0,844 & & \\
\hline & HUDD3 & 0,822 & & \\
\hline & HUDD1 & 0,799 & & \\
\hline \multirow{3}{*}{ Neo quyết định } & NQD1 & 0,855 & \multirow{3}{*}{0,855} & \multirow{3}{*}{0,709} \\
\hline & NQD2 & 0,850 & & \\
\hline & NQD3 & 0,837 & & \\
\hline \multirow{4}{*}{ Quyết định đầu tư } & QDDT4 & 0,828 & \multirow{4}{*}{0,865} & \multirow{4}{*}{0,694} \\
\hline & QDDT2 & 0,818 & & \\
\hline & QDDT1 & 0,815 & & \\
\hline & QDDT3 & 0,799 & & \\
\hline \multirow{4}{*}{ Hiệu quả đầu tư } & HQDT1 & 0,875 & \multirow{4}{*}{0,908} & \multirow{4}{*}{0,740} \\
\hline & HQDT4 & 0,872 & & \\
\hline & HQDT2 & 0,842 & & \\
\hline & HQDT3 & 0,835 & & \\
\hline \multirow{2}{*}{\multicolumn{2}{|c|}{$\begin{array}{l}\text { Bên cạnh, để đánh giá tính giá trị của } \\
\text { thang đo trong nghiên cứu, Vuong và } \\
\text { Suntrayuth }(2020) \text { đã đề xuất sử dụng hệ số } \\
\text { nhân tố khám phá để đo lường giá trị này. } \\
\text { Hệ số tải nhân tố nên lớn hơn } 0,5 \text { được xem } \\
\text { là có ý nghĩa thực tiễn. Như thể hiện trong } \\
\text { Bảng } 2 \text {, hầu hết các giá trị của hệ số tải của } \\
\text { mối chỉ số đều cao hơn } 0,5 \text { (giá trị bé nhất là }\end{array}$}} & \multicolumn{3}{|c|}{$\begin{array}{l}\text { 0,723). Do đó, mỗi cấu trúc thể hiện giá trị } \\
\text { hội tụ tôt. }\end{array}$} \\
\hline & & \multicolumn{3}{|c|}{$\begin{array}{l}\text { Từ các kết quả phân tích ở trên, } \\
\text { chúng ta có thể kết luận rằng các thang đo } \\
\text { được sử dụng trong mô hình nghiên cứu đã } \\
\text { đạt được độ tin cậy và tính giá trị rất tốt. Do } \\
\text { đó, các thang đo này sẽ sử dụng để phân tích } \\
\text { trong mô hình hồi quy bội. }\end{array}$} \\
\hline
\end{tabular}




\subsection{Phân tích tương quan}

Trước khi tiến hành phân tích hồi quy, tác giả sử dụng hệ số tương quan Pearson để lượng hóa mức độ chặt chẽ của mối liên hệ tuyến tính giữa hai biến độc lập và phụ thuộc (Vuong \& Giao, 2020). Trong phân tích tương quan Pearson, không có sự phân biệt giữa biến độc lập và biến phụ thuộc mà tất cả các biến đều được xem xét như nhau. Tuy nhiên, nếu các biến có tương quan chặt thì phải lưu ý đến vấn đề đa cộng tuyến sau khi phân tích hồi quy.

\section{Bảng 3. Kết quả phân tích tương quan}

\begin{tabular}{|c|c|c|c|c|c|c|c|c|}
\hline & & QTT & KHSC & THDH & HUDD & NQD & QDDT & HQDT \\
\hline \multirow{3}{*}{ QTT } & Pearson Correlation & 1 &, $364^{* * *}$ &,- 022 &, $213^{* *}$ &, $330^{* *}$ &, $387^{* *}$ &,$- 419^{* *}$ \\
\hline & Sig. (2-tailed) & &, 000 & ,658 & ,000 &, 000 &, 000 &, 000 \\
\hline & $\mathrm{N}$ & & 411 & 411 & 411 & 411 & 411 & 411 \\
\hline \multirow{3}{*}{ KHSC } & Pearson Correlation & & 1 & ,008 &, $329^{* *}$ &, $370^{* *}$ &, $381^{* *}$ &,$- 496^{* *}$ \\
\hline & Sig. (2-tailed) & & & ,871 & ,000 & ,000 & ,000 &, 000 \\
\hline & $\mathrm{N}$ & & & 411 & 411 & 411 & 411 & 411 \\
\hline \multirow{3}{*}{ THDH } & Pearson Correlation & & & 1 &,- 068 & ,034 & ,082 &,$- 112^{*}$ \\
\hline & Sig. (2-tailed) & & & & , 170 & ,492 & ,098 & ,023 \\
\hline & $\mathrm{N}$ & & & & 411 & 411 & 411 & 411 \\
\hline \multirow{3}{*}{ HUDD } & Pearson Correlation & & & & 1 &, $262^{* *}$ &, $288^{* *}$ &,$- 508^{* * *}$ \\
\hline & Sig. (2-tailed) & & & & & ,000 & ,000 & ,000 \\
\hline & $\mathrm{N}$ & & & & & 411 & 411 & 411 \\
\hline \multirow{3}{*}{ NQD } & Pearson Correlation & & & & & 1 &, $495^{* *}$ &,$- 519^{* *}$ \\
\hline & Sig. (2-tailed) & & & & & & ,000 &, 000 \\
\hline & $\mathrm{N}$ & & & & & & 411 & 411 \\
\hline \multirow{3}{*}{ QDDT } & Pearson Correlation & & & & & & 1 &,$- 498^{* *}$ \\
\hline & Sig. (2-tailed) & & & & & & &, 000 \\
\hline & $\mathrm{N}$ & & & & & & & 411 \\
\hline \multirow{3}{*}{ HQDT } & Pearson Correlation & & & & & & & 1 \\
\hline & Sig. (2-tailed) & & & & & & & \\
\hline & $\mathrm{N}$ & & & & & & & \\
\hline
\end{tabular}

Ghi chú: ** p <0.01; * p <0.05

Xem xét ma trận tương quan (Bảng 3) cho thấy rằng mức ý nghĩa của hầu hết các hệ số rất nhỏ $(\mathrm{sig}=0<0,05)$ nên hầu hết các hệ số tương quan có ý nghĩa thống kê và đều đủ điều kiện để đưa vào phân tích hồi quy.

\subsection{Phân tích hồi quy}

Trên cơ sở thang đo các yếu tố ảnh hưởng đến Quyết định đầu tư và hiệu quả đầu tư đã được xem xét mối tương quan tuyến tính, tiếp tục sử dụng phân tích hồi quy để thấy mối quan hệ giữa các nhân tố này.

Đánh giá đa cộng tuyến là bước đầu tiên trong phân tích mô hình hồi quy. Quy trình này là cần thiết để đảm bảo rằng các hệ số được ước tính bằng cách hồi quy các biến nội sinh trên các biến ngoại sinh đính kèm không bị sai lệch. Trong hồi quy, tính đa cộng tuyến tồn tại khi hai hoặc nhiều biến độc lập có tính tương quan cao. Đa cộng tuyến trong hồi quy làm tăng sai số chuẩn, làm cho các kiểm định quan trọng của các biến độc lập không đáng tin cậy. Tính đa cộng tuyến cao làm suy yếu khả năng của nhà nghiên cứu để đưa ra phán đoán về tầm quan trọng tương đối của một biến độc lập so với biến khác (Giao \& Vương, 2019). Do đó, khi tính đa cộng tuyến cao, nhà nghiên cứu phải tránh phân tích so sánh về tầm quan trọng của chỉ số dựa trên trọng số đường dẫn. Theo Giao và Vương (2019), đa cộng tuyến có thể xảy ra nếu hệ số phương sai phóng đại (VIF) vượt quá 5 . Theo bảng Bảng 4, hệ số VIF đạt giá trị lớn nhất 1,326 (nhỏ hơn 5) cho thấy các biến độc lập này 
không có quan hệ chặt chẽ với nhau nên

không có hiện tượng đa cộng tuyến xảy ra.

Bảng 4. Bảng tổng kết quả phân tích hồi quy của 3 mô hình

\begin{tabular}{|c|c|c|c|c|c|}
\hline Biến & $\begin{array}{l}\text { Mô hình } 1 \\
\text { Quyết định } \\
\text { đầu tư } \\
\end{array}$ & $\begin{array}{l}\text { Mô hình } \\
2 \\
\text { Hiệu ql }\end{array}$ & $\begin{array}{c}\text { Mô hình } \\
3 \\
\text { ả đầu tư }\end{array}$ & Hệ số VIF & Kết luận \\
\hline Sự quá tự tin & $0,199 * * *$ & & & 1,222 & Chấp nhận \\
\hline Khuynh hướng sẵn có & $0,143 * * *$ & & & 1,326 & Chấp nhận \\
\hline $\begin{array}{l}\text { Khuynh hướng đại } \\
\text { diện }\end{array}$ & $0,081 *$ & & & 1,009 & Chấp nhận \\
\hline Hiệu ứng đám đông & $0,114 * *$ & & & 1,164 & Chấp nhận \\
\hline Neo quyết định & $0,344 * * *$ & & & 1,249 & Chấp nhận \\
\hline Quyết định đầu tư & & $-0,498 * * *$ & & 1,000 & Chấp nhận \\
\hline Giới tính & & & $0,103^{*}$ & 1,019 & Chấp nhận \\
\hline Tuổi & & & $-0,168 * *$ & 1,029 & Chấp nhận \\
\hline Trình độ & & & 0,064 & 1,061 & Bác bỏ \\
\hline Kinh nghiệm đầu tư & & & $0,128 *$ & 1,038 & Chấp nhận \\
\hline $\begin{array}{l}\text { Mức độ giải thích của } \\
\text { mô hình }\left(\mathrm{R}^{2} \text { hiệu }\right. \\
\text { chỉnh })\end{array}$ & 0,633 & 0,246 & 0,045 & & \\
\hline
\end{tabular}

Ghi chú: ***: mức ý nghĩa $<0.0001 ; * *$ : mức ý nghĩa $<0.001 ; *$ : mức ý nghĩa $<0.05$

Tiếp theo, nghiên cứu đã xem xét đến độ phù hợp tổng thể của mô hình. Theo Vuong và cộng sự. (2020), chất lượng của mô hình hồi quy bộ nên được đánh giá bằng chỉ số mức độ phù hợp mô hình $\mathrm{R}^{2}$ hiệu chỉnh. Những tác giả này cũng đã đề xuất rằng hệ số $\mathrm{R}^{2}$ hiệu chỉnh có giá trị trên 0,26 cho thấy chất lượng mô hình ảnh hưởng lớn; dao động từ 0,13 đến 0,26 cho ảnh hưởng trung bình và dưới 0,02 cho ảnh hưởng nhỏ. Bảng 4 cho thấy rằng, giá trị $\mathrm{R}^{2}$ hiệu chỉnh của mô hình 1 bằng 0,633 có nghĩa là $63,3 \%$ sự biến thiên của biến QDDT (Quyết định đầu tư) được giải thích bởi sự biến thiên của 05 biến độc lập QTT, KHSC, THDH, HUDD, NQD. Bên cạnh đó, giá trị $\mathrm{R}^{2}$ hiệu chỉnh của mô hình 2 và 3 lần lượt là 0,246 và 0,045 . Do đó, chúng ta có thể kết luận rằng, chất lượng mô hình hồi quy 1 có mức ảnh hưởng cao, chất lượng mô hình hồi quy
2 có mức ảnh hưởng trung bình và mức độ ảnh hưởng yếu đối với mô hình hồi quy 3 .

Dựa vào kết quả phân tích hồi quy bội (Bảng 4), mô hình hồi quy 1 cho thấy 05 biến độc lập NQD, THDH, HUDD, QTT, KHSC có tác động cùng chiều vào biến phụ thuộc quyết định đầu tư vì hệ số hồi quy chuẩn hóa $(\beta)$ của các biến này đều dương và có ý nghĩa thống kê (Sig.<0,05). So sánh mức độ tác động của 05 biến này vào biến phụ thuộc quyết định đầu tư (QDDT) theo thứ tự giảm dần như sau: ta thấy biến $\mathrm{Neo}$ quyết định (NQD) có tác động mạnh nhất $\left(\beta_{5}=0,344\right)$, tiếp theo là biến Sự quá tự tin (QTT) $\left(\beta_{1}=0,199\right)$, tiếp đến là biến Khuynh hướng sã̃n có (KHSC) $\left(\beta_{2}=0,143\right)$, tiếp đến là biến Hiệu ứng đám đông (HUDD) $\left(\beta_{4}=\right.$ $0,114)$, và tác động thấp nhất là biến Tình huống điển hình $(\mathrm{THDH})\left(\beta_{3}=0,081\right)$. Bên cạnh đó, mô hình hồi quy 2 cho thấy rằng biến Quyết định đầu tư (do thúc đẩy bởi các 
biến tài chính hành vi) đã có tác động ngược chiều vào biến phụ thuộc hiệu quả đầu tư vì hệ số hồi quy chuẩn hóa ( $\beta$ ) của biến này âm $(\beta=-0,498)$ và có ý nghĩa thống kê (Sig.<0,05). Như vậy các giả thuyết $\mathrm{H}_{1}, \mathrm{H}_{2}$, $\mathrm{H}_{3}, \mathrm{H}_{4}, \mathrm{H}_{5}, \mathrm{H}_{6}$ đều được chấp nhận ở độ tin cậy $95 \%$.

Cuối cùng, từ bảng thống kê phân tích các hệ số hồi quy 3 , kết quả nghiên cứu đã cho thấy rằng giới tính, kinh nghiệm đầu tư đã ảnh hưởng cùng chiều đến hiệu quả đầu tư $(\beta=0,103 ; 0,128$, và Sig. $<0,05)$. Nói cách khác, những nhà đầu tư nam có kinh nghiệm đầu tư có khuynh hướng thu được hiệu quả đầu tư cao hơn những nhà đầu tư nữ ít kinh nghiệm. Bên cạnh đó, nghiên cứu cũng đã chỉ ra rằng nhửng nhà đầu tư càng lớn tuổi có khuynh hướng đầu tư ít hiệu quả hơn các nhà đầu tư trẻ tuổi $(\beta=-0.168$, và $\mathrm{Sig}$. < $0,05)$. Tuy nhiên, nghiên cứu này chưa tìm thấy mối quan hệ giữa trình độ học vấn và hiệu quả đầu tư của các nhà đầu tư cá nhân tại thị trường chứng khoán TP. HCM ( $\beta=$ 0,064 , và Sig. > 0,05).

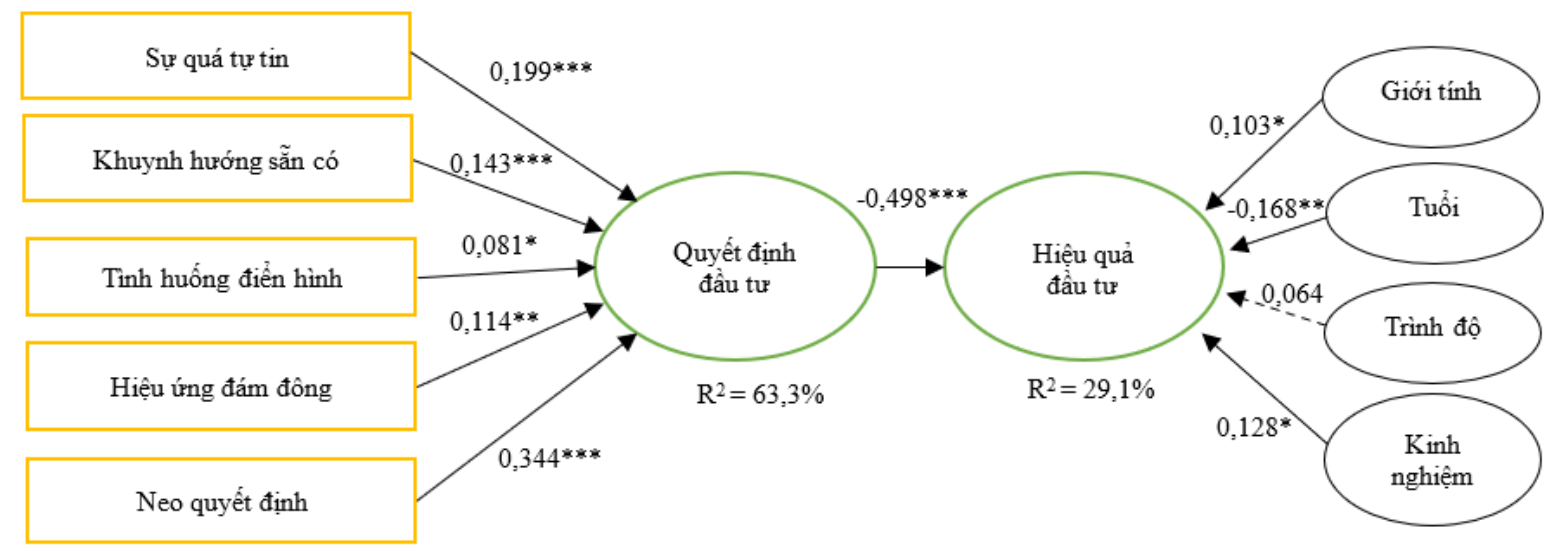

Hình 3. Mô hình kết quả nghiên cứu

\section{KẾT LUẬN VÀ HÀM Ý QUẢN TR!}

\subsection{Kết luận}

Căn cứ vào tổng quan lý thuyết, mô hình nghiên cứu đã được phát triển cho nghiên cứu này. Mô hình này đã được kiểm tra với một mẫu gồm 421 nhà đầu tư cá nhân tại thị trường chứng khoán TP. HCM. Với những kết quả thu được, nghiên cứu này có những đóng góp tích cực trong thực tiễn quản lý, cụ thể như sau: Về thang đo sử dụng trong nghiên cứu này. Kết quả nghiên cứu cho thấy rằng toàn bộ thang đo được sử dụng trong nghiên cứu là đáng tin cậy (Cronbach's Alpha $>0,8$ ) và có thể được sử dụng cho những nghiên cứu khác. Về các yếu tố ảnh hưởng tài chinh hành vi đến quyết định đầu tư và hiệu quả đầu tư, nghiên cứu đã chỉ ra rằng có 05 thành phần của tài chinh hành vi tác động đến quyết định đầu tư từ mạnh nhất đến thấp nhất theo thứ tự sau: Neo quyết định, Sự quá tự tin, Khuynh hướng sẵn có, Hiệu ứng đám đông, Tình huống điển hình. Ngoài ra, quyết định đầu tư đã ảnh hưởng tiêu cực đến hiệu quả đầu tư (Hình 3).

\subsection{Hàm ý quản trị}

\section{Nhóm yếu tố "Neo quyết định"}

Kết quả nghiên cứu cho thấy "Neo quyết định" là yểu tố có mức độ ảnh hưởng tích cực lớn nhất đến quyết định đầu tư $\left(\beta_{5}=\right.$ $0,344)$ và từ đó làm giảm đi hiệu quả đầu tư. Để gia tăng hiệu quả đầu tư của nhà đầu tư cá nhân thông qua yếu tố này, các nhà đầu tư nên suy nghĩ rằng "bạn có vội vã trong các quyết định trong quá khưo"- bởi vì khi bạn neo các quyết định của mình cho một mốc nào đó trong quá khứ, thì hãy xem lại cái mốc đó có đáng tin cậy không. Sau đó hãy dành thời gian để đưa ra các quyết định chậm hơn và sẵn sàng giữ nguyên quan 
điểm như vậy nếu bạn vẫn cảm thấy áp lực cho việc đưa ra các quyết định của mình. Nếu một người nào đó gây áp lực với bạn cho một quyết định, điều đó có thể là dấu hiệu cho thấy những điều đó chống lại lợi ích tốt nhất của bạn.

\section{Nhóm yếu tố "Sự quá tụ tin"}

Kết quả nghiên cứu cho thấy "Sự quá tự tin" là yếu tố có mức độ ảnh hưởng tích cực mạnh thứ hai đến quyết định đầu tư $\left(\beta_{1}\right.$ $=0,199$ ) và từ đó làm giảm đi hiệu quả đầu tư. Để gia tăng hiệu quả đầu tư của nhà đầu tư cá nhân thông qua yếu tố này, các nhà đầu tư nên giao dịch ít hơn và đầu tư nhiều hơn. Hay từ bỏ suy nghĩ rằng làm sao bạn có thế nghĩ mình thông minh hơn máy tính, các nhà đầu tư tổ chức, các quỹ đầu tư khi họ có nhiều kinh nghiệm và dữ liệu hơn, bỏ qua những suy nghĩ những thông tin của mình có là tốt hơn người khác. Khi nhà đầu tư nắm và thực hiện được thông điệp trên họ sẽ đầu tư một cách hiểu quả hơn.

\section{Nhóm yếu tố "Khuynh hướng sã̃n có"}

Kết quả nghiên cứu cho thấy "Khuynh hướng sẵn có" là yếu tố có mức độ ảnh hưởng tích cực mạnh thứ ba đến quyết định đầu tư $\left(\beta_{2}=0,143\right)$ và từ đó làm giảm đi hiệu quả đầu tư. Để gia tăng hiệu quả đầu tư của nhà đầu tư cá nhân thông qua yếu tố này, các nhà đầu tư nên sử dụng Phương pháp phân tích kỹ thuật như là một cơ sở chủ yểu trước khi họ đưa ra các quyết định đầu tư. Tự trau dồi các kiến thức về phân tích chứng khoán: Để có thể thành công trên thị trường này, các kiến thức cơ bản về kinh tế vĩ mô, các nhà đầu tư phải nắm rõ ràng, tác động của các yếu tố lạm phát, lãi suất, tỷ giá, cung tiền, nguồn vốn... là các yếu tố ảnh hưởng trực tiếp đến thị trường. Thường xuyên tìm tòi, nghiên cứu các tài liệu để mối quan hệ giữa các biến này, để có thể nhìn nhận thị trường một cách toàn diện và nhanh nhạy hơn. Bên cạnh đó một nhà đầu tư cá nhân phải trang bị kiến thức phân tích cơ bản và kỹ thuật như là công cụ chính trong đầu tư tài chính của mình. Một thực tế cho thấy các nhà đầu tư Việt Nam chú trọng vào phân tích kỷ thuật quá nhiều, bỏ qua các yếu tố cơ bản. Nâng cao kỹ năng phân tích cơ bản và kết hợp phân tích kỹ thuật có đầu tư một cách thông minh hơn. Ngoài ra, các nhà đầu tư cá nhân cần phải trau dồi kinh nghiệm đầu tư thường xuyên, điều đó giúp các nhà đầu tư tìm được hướng giải quyết trong các tình huống khó khăn. Bên cạnh đó là học tập các kinh nghiệm của các nhà đầu tư nước ngoài, tham khảo và tìm cho mình một chiến lược đầu tư phù hợp với bản thân.

\section{Nhóm yếu tố "Hiệu ứng đám đông"}

Kết quả nghiên cứu cho thấy "Hiệu ứng đám đông" là yếu tố có mức độ ảnh hưởng tích cực mạnh thứ tư đến quyết định đầu tư $\left(\beta_{4}=0,114\right)$ và từ đó làm giảm đi hiệu quả đầu tư. Để gia tăng hiệu quả đầu tư của nhà đầu tư cá nhân thông qua yếu tố này, các nhà đầu tư hãy xem xét rằng các thông tin truyền thông không phải là báo hiệu của sự khôn ngoan, mà nơi gây ra các cảm xúc, cố gắng vứt bỏ và nhìn về phía trước, hãy xây dựng một kế hoạch dài hơn với các nguyên tác đầu tư mạnh mẽ, tránh các làn sóng ngắn hạn, nên đầu tư thời gian để trang bị các kiến thức đầy đủ, các quyết định đầu tư dựa vào các phân tích có căn cứ và suy luận rõ ràng dựa trên các thông tin có được. Từ đó tạo ra cho họ những lối suy nghĩ riêng, không cuốn theo hành vi của đám đông. Tuy nhiên, nếu cứng nhắc quá với các suy luận của mình, quá tự tin vào khả năng và bảo thủ với các quan điểm đó cũng là một sai lầm dẫn đến các quyết định không kịp thời có thể đánh mất co hội hoặc không thoát khởi vùng nguy hiểm.

\section{Nhóm yếu tố "Tình huống điển hình"}

Kết quả nghiên cứu cho thấy "Tình huống điển hình" là yếu tố có mức độ ảnh hưởng tích cực yếu nhất đến quyết định đầu tư $\left(\beta_{3}=0,081\right)$ và từ đó làm giảm đi hiệu quả đầu tư. Để gia tăng hiệu quả đầu tư của nhà đầu tư cá nhân thông qua yếu tố này, các nhà đầu tư cá nhân tại thị trường chúng khoán TP. HCM còn chịu ảnh hưởng bởi tình huống điển hình. Họ chịu ảnh hưởng bởi các yếu tố (sự thay đổi) gần đây để đánh 
giá, dự đoán sự thay đổi giá trong tương lai, hay neo các quyết định của mình với tham chiếu ban đầu. Những trải nghiệm của họ trong quá khứ là một bài học đáng giá, tuy nhiên với tầm quan trọng của các nhân tố này các nhà đầu tư phải xem xét thận trọng trong quá trình đưa ra quyết định đầu tư và một lần nữa hãy đưa đảm bảo các quyết định của mình được xem xét từ nhiều khía cạnh. Hơn nữa, việc sử dụng các thông tin một cách dễ dàng khiến các hành vi đó làm họ thất bại. Do đó cần nâng cao khả năng sàng lọc các luồng thông tin mình nhận được trong một thị trường còn nhiều khe hở ở hành lang pháp lý. Để làm được điều này, các nhà đầu tư phải có kiến thức về phân tích và đầu tư chứng khoán, biết lựa chọn thông tin chính thống, biết cách tiếp cận nguồn thông tin từ đó tự đưa ra các phân tích cá nhận để lựa chọn thông tin tránh tiếp nhận thông tin một cách dễ dàng và hành động thiếu suy nghĩ.

\section{HAN CHẾ CỦA ĐỀ TÀI VÀ HƯỚNG NGHIÊN CÚUU TIẾP THEO}

Bên cạnh những mặt đạt được nêu trên, đề tài chỉ ra một số hạn chế còn tồn tại như sau: Thứ nhất, nghiên cứu chỉ được thực hiện với các đối tượng khảo sát là các nhà đầu tư cá nhân tại thị trường chứng khoán HOSE với phương pháp lấy mẫu thuận tiện, do đó kết

\section{TÀI LIỆU THAM KHẢO}

Abul, S. J. (2019). Factors influencing Individual Investor Behaviour: Evidence from the Kuwait Stock Exchange. Asian Social Science, 15(3), 27-39.

Anderson, A., Henker, J., \& Owen, S. (2005). Limit order trading behavior and individual investor performance. Journal of Behavioral Finance, 6(2), 71-89.

Bakar, S., \& Yi, A. N. C. (2016). The impact of psychological factors on investors' decision making in malaysian stock market: A case of Klang Valley and Pahang. Procedia quả nghiên cứu mang tính đại diện chưa cao. Vì vậy, nghiên cứu tiếp theo nên sử dụng phương pháp lấy mẫu xác suất để tăng tính đại diện. Bên cạnh đó, các nghiên cứu sau nên mở rộng với một kích thước mẫu lớn hơn, đa dạng các nhà đầu tư, không chỉ là các nhà đầu tư giao dịch tại sở giao dịch $\mathrm{Tp}$. Hồ Chí Minh mà còn giao dịch tại Sở giao dịch chứng khoán Hà Nội. Các nghiên cứu nên tăng cường đo lường tài chính hành vi cũng như tìm ra được mô hình phù hợp hơn cho thị trường chứng khoán Việt Nam. Thứ hai, nghiên cứu này chỉ tập trung khảo sát các nhà đầu tư cá nhân, tác giả đề xuất nên mở rộng cho các nhà đầu tư tổ chức và các tổ chức tham gia thị trường để khám phá các hành vi tồn tại trên thị trường và đưa ra các giải pháp tốt hơn cho cho các thành phần tham gia thị trường và nhà quản lý. Thứ ba, nghiên cứu này chỉ giải thích được $63,3 \%$ sự biến thiên của quyết định đầu tư bởi bởi sự biến thiên của 05 biến tài chính hành vi. Như vậy, còn nhiều yếu tố khác ảnh hưởng đến quyết định đầu tư mà nghiên cứu chưa tìm ra. Vì vậy, nghiên cứu tiếp theo cần nghiên cứu sâu hơn để tìm ra một số yếu tố tài chính hành vi mới ảnh hưởng đến quyết định đầu tư, để từ đó có thể nâng cao hiệu quả đầu tư.

Economics and Finance, 35(3), 319328.

Barberis, N., \& Thaler, R. (2003). A Survey of Behavioral Finance. In G. M. Constantinides, M. Harris, \& R. M. Stulz (Eds.), Handbook of the Economics of Finance (Vol. 1, Part 2, pp. 1053-1128): Elsevier.

Bikas, E., Jurevičienè, D., Dubinskas, P., \& Novickytè, L. (2013). Behavioural Finance: The Emergence and Development Trends. Procedia Social and Behavioral Sciences, 82(5), 870-876.

Cen, L., Hilary, G., \& Wei, K. C. J. (2013). The role of anchoring bias in the equity market: Evidence from analysts' earnings forecasts and 
stock returns. Journal of Financial and Quantitative Analysis, 48(7), 4776.

Chen, G., Kim, K. A., Nofsinger, J. R., \& Rui, O. M. (2017). Trading performance, disposition effect, overconfidence, representativeness bias, and experience of emerging market investors. Journal of Behavioral Decision Making, 20(4), 425-451. doi:10.1002/bdm.561

Chiang, T. C., \& Zheng, D. (2010). An empirical analysis of herd behavior in global stock markets. Journal of Banking \& Finance, 34(8), 19111921.

doi:https://doi.org/10.1016/j.jbankfin .2009.12.014

De Bondt, W. F. M., \& Thaler, R. (1985). Does the stock market overreact? The Journal of Finance, 40(3), 793805.

Dima, W., Al-Abdallah, S., \& Abualjarayesh, N. (2018). Behavioral biases and investment performance: Does gender matter? Evidence from amman stock exchange. Jordan Journal of Economic Sciences, 5(1), 77-92.

Fama, E. F. (1998). Market efficiency, longterm returns, and behavioral finance. Journal of Financial Economics, 49(3), 283-306.

Fernandes, J., Peña, J. I., \& Tabak, B. (2007). Behavior Finance and Estimation Risk in Stochastic Portfolio Optimization. Applied Financial Economics, 20(9), 1-61.

Giao, H. N. K., \& Vương, B. N. (2019). Giáo Trình Cao Hoc Phuong Pháp Nghiên Cưu Khoa Học Trong Kinh Doanh Cập Nhật SmartPLS. TP. Hồ Chí Minh, Việt Nam: Nhà Xuất Bản Tài Chính.

Greenberg, A. E., \& Hershfield, H. E. (2019). Financial decision making. Consumer Psychology Review, 2(1), 17-29. doi:10.1002/arcp.1043
HOSE. (2010). A Look Back on 10 Years of Development. Ho Chi Minh: The Ho Chi Minh Stock Exchange.

Kahneman, D., \& Tversky, A. (1979). Prospect Theory: An Analysis of Decision under Risk. Econometrica, 47(2), 263-291.

Kengatharan, L., \& Navaneethakrishnan, K. (2014). The Influence of Behavioral Factors in Making Investment Decisions and Performance: Study on Investors of Colombo Stock Exchange, Sri Lanka. Asian Journal of Finance \& Accounting, 6(2), 1-24. doi:10.5296/ajfa.v6i1.4893

Kim, K., \& Nofsinger, J. (2008). Behavioral finance in Asia. Pacific-Basin Finance Journal, 16(1), 1-7.

Kourtidis, D. (2017). The role of personality traits in investors trading behaviour: empirical evidence from Greek. International Journal of Social Economics, 44(11), 1402-1420. doi:10.1108/IJSE-07-2014-0151

Longjie, X., \& Anfeng, Z. (2017). The impact of managers overconfidence on corporate investment. International Journal of Social Science and Humanity, 7(2), 109114.

Maditinos, D. I., Sevic, Z., \& Theriou, N. G. (2017). Investors' behavior in the Athens Stock Exchange (ASE). Studies in Economics and Finance, 24(1), 32-50.

Markowitz, H. (1952). Portfolio selection. The Journal of Finance, 7(1), 77-91.

Masomi, S. R., \& Ghayekhloo, S. (2011). Consequences of human behaviors'in economic: The effects of behavioral factors in investment decision making at Tehran Stock Exchange International Conference on Business and Economics Research, 1(2), 234 - 237.

Odean, T. (1998). Volume, Volatility, Price, and Profit When All Traders Are Above Average. The Journal of 
Finance, $\quad$ 53(6), 1887-1934. doi:10.1111/0022-1082.00078

Olsen, R. A. (1998). Behavioral Finance and Its Implications for Stock-Price Volatility. Financial Analysts Journal, 54(2), 10-18.

Qamruzzaman, M., \& Wei, J. (2018). Financial innovation, stock market development, and economic growth: An application of ardl model. International Journal of Financial Studies, 6(3), 69-99.

Sharpe, W. F. (1964). The Sharpe Ratio. The Journal of Portfolio Management, 21(1), 49. doi:10.3905/jpm.1994.409501

Shefrin, H., \& Statman, M. (2000). Behavioral Portfolio Theory. The Journal of Financial and Quantitative Analysis, 35(2), 127151.

Shiller, R. J. (2005). Irrational Exuberance (2nd Ed.). Princeton, NJ: Princeton University Press.

Shleifer, A., \& Vishny, R. W. (2017). A Survey of Corporate Governance. The Journal of Finance, 52(2), 737$783 . \quad$ doi:10.1111/j.15406261.1997.tb04820.x

Singha, M. M., \& Sunita, M. (2015). Behavioral finance: A study on gender based dilemma in making investment decisions. Sumedha Journal of Management, 4(1), 4-16.

Stockbiz. (2019). Thống kê thị trường. Retrieved from https://www.stockbiz.vn/MarketMov ers.asp $x$ ?Exchange $=$ HOSTC

Vndirect. (2019). Biểu đồ kỹ thuật. Retrieved from https://www.vndirect.com.vn/portal/ bieu-do-ky-thuat/vnindex.shtml

Vuong, B. N., \& Giao, H. N. K. (2020). The impact of perceived brand globalness on consumers' purchase intention and the moderating role of consumer ethnocentrism: An evidence from
Vietnam. Journal of International Consumer Marketing, 32(1), 47-68. doi:10.1080/08961530.2019.161911 5

Vuong, B. N., Hieu, V. T., \& Trang, N. T. T. (2020). An empirical analysis of mobile banking adoption in Vietnam. Gestão e Sociedade, 14(1), 33653393

doi:https://doi.org/10.21171/ges.v14i 37.3078

Vuong, B. N., \& Suntrayuth, S. (2020). The impact of human resource management practices on employee engagement and moderating role of gender and marital status: An evidence from the Vietnamese banking industry. Management Science Letters, 10(7), 1633-1648. doi:https://doi.org/10.5267/j.msl.201 9.12.003

Waweru, N. M., Munyoki, E., \& Uliana, E. (2008). The effects of behavioural factors in investment decisionmaking: a survey of institutional investors operating at the Nairobi Stock Exchange. International Journal of Business and Emerging Markets, 1(1), 24-41.

Weber, E. U., \& Hsee, C. K. (2010). Culture and individual judgment and decision making. Applied Psychology: An International Review, 49(1), 32-61. doi:10.1111/1464-0597.00005

Yates, J. F., Lee, J.-W., \& Bush, J. G. (1997). General knowledge overconfidence: Cross-national variations, response style, and reality. Organizational Behavior and Human Decision Processes, 70(2), 87-94. doi:10.1006/obhd.1997.2696

Zuravicky, O. (2005). The Stock Market: Understanding And Applying Ratios, Decimals, Fractions, And Percentages. New York: Powerkids Pr. 